\title{
In Silico Identification of B-Cell Epitopes of Leishmania infantum Recombinant Histone Shared with Human Sera Stably Living in Area Where Leishmania Species Does Perpetuate
}

\author{
Sami Lakhal ${ }^{1^{*}}$ and Malcolm S Duthie ${ }^{2}$ \\ ${ }^{1}$ Laboratory of Virology, Veterinary Research Institute of Tunisia, Rue Djebel Lakhdhar-La Rabta 1006 Tunis, Tunisia \\ ${ }^{2}$ Infectious Disease Research Institute, Suite 400, 1616 Eastlake Ave E, Seattle, WA 98102, USA
}

"Corresponding author: Sami Lakhal, Laboratory of Virology, Veterinary Research Institute of Tunisia, Rue Djebel Lakhdhar-La Rabta 1006 Tunis, Tunisia, Tel: (+216)71564321; E-mail: samilakh@aol.com

Rec date: Apr 24, 2017; Acc date: Jun 13, 2017; Pub date: Jun 15, 2017

Copyright: ( 2017 Lakhal S, et al. This is an open-access article distributed under the terms of the creative commons attribution license, which permits unrestricted use, distribution, and reproduction in any medium, provided the original author and source are credited.

\begin{abstract}
Visceral leishmaniasis $(\mathrm{VL})$ can initially be misdiagnosed because its presentation is similar to many autoimmune diseases such as systemic lupus erythematosus (SLE), autoimmune hepatitis and dermatomyositis. Furthermore, serum antibodies from VL patients have been shown to strongly react against proteins that are conserved between the causative agent, $L$. infantum, and humans themselves. Some of these proteins, like histone, have also been described as immunogenic in several auto-immune syndromes, and the detection of antibodies against them is considered to be indicative of immune system disorders.

The potential overlap of autoimmune diseases and VL presents a situation of confounding diagnoses if crossreactive tests are used. To explore this possibility, we screened sera from three Tunisian populations for the presence, and relative quantity, of antibodies against a panel of $L$. infantum antigens comprising crude extract or recombinant molecules, with special attention being given to evolutionarily conserved histones. Our data indicate that antibodies in many of the SLE at-risk individuals recognized crude soluble Leishmania antigen (SLA). This compromised the specificity of SLA-based ELISA, providing many results falsely indicating $L$. infantum infection. Examination of the crude Leihsmania histone (CLH) mixture, which is expected to contain nucleosomal Leishmania histones $\mathrm{H} 2 \mathrm{~A}, \mathrm{H} 2 \mathrm{~B}$ and $\mathrm{H} 4$, as well as recombinant versions of these Leishmania histones, suggested these to be a source of the cross-reactivity. For the purposes of diagnosing $V L$, it is therefore important to note that the rK39 antigen was found to be more specific and not conflicting with autoimmune presentations. In silico prediction data validate and indicate that the human histones are immunologically cross-reactive with Leishmania histones.
\end{abstract}

Keywords: Systemic lupus erythematosus; Associated autoantigen panels; Autoantibodies; Leishmania histones; Autoimmune diseases

\section{Introduction}

Visceral leishmaniasis (VL) is a severe, life-threatening neglected tropical disease caused by infection with the vector-borne parasites Leishmania donovani and $L$. infantum. The vast majority of cases are reported in developing countries and VL is considered to be a disease of poverty [1]. In Europe, Central Asia and North Africa and West, $L$. infantum species are responsible for VL. L. infantum infection may be asymptomatic (subclinical), or may emerge to cause VL [2]. The disease occurs mainly in young people and in immuno-compromised individuals $[3,4]$. VL diagnosis can be attained directly through the visualization of parasites by microscopic examination in spleen or bone marrow cell aspirates, and complemented by detection of $L$. infantum DNA through polymerase chain reaction (PCR)-based assays in the blood [5]. Alternatively, diagnosis can be reached by detection of parasite-specific antibodies in the enzyme-linked immunosorbent assay (ELISA)-based [5,6]. While some $L$. infantum antigenic proteins have emerged as signatures of asymptomatic parasitism, others such as the recombinant kinesin 39 (rK39) have emerged as an indicator of active disease. Indeed, rK39 has proven to be a very sensitive and specific antigen for the sero-diagnosis of VL [7-10].
In our previous studies, in addition to soluble Leishmania antigens (SLA), we identified evolutionarily conserved Leishmania histones as having potential to complement antibody-based tests for both human and canine VL. These proteins were identified using sera from VLaffected dogs [11-14], with immunoscreening identifying antibodies binding $L$. infantum histone $\mathrm{H} 2 \mathrm{~A}$, as well as $\mathrm{H} 2 \mathrm{~B}$ and $\mathrm{H} 4$ [14] that belong to the nucleosomal core of $L$. infantum. In addition, antibodies against recombinant $(\mathrm{r}) \mathrm{H} 2 \mathrm{~A}$ and $\mathrm{rH} 2 \mathrm{~B}$ were detected in the sera of many Tunisian VL patients $[8,15]$. VL patients presenting with clinical and laboratory features that mimic autoimmune hepatitis, primary biliary cirrhosis $[16,17]$ and systemic lupus erythematosus (SLE), could therefore be mistakenly misdiagnosed. Like rheumatic disease Leishmania-infected patients have higher level of circulating immune complexes and autoantibodies like rheumatoid factor and hypergammaglobulinemia and higher titers of antinuclear antibodies (ANA) observed in systemic autoimmune diseases in particular SLE [18-20].

Apparently healthy subjects at risk of developing autoimmune diseases reside in areas within Tunisia where L. infantum and bloodfeeding phlebotomine sandflies are endemic, thereby also presenting risk for infection and development of VL [21]. To explore this, we screened sera from three Tunisian populations for the presence, and relative quantity, of antibodies against a panel of $L$. infantum antigens comprising crude extract or recombinant molecules, with special attention being given to evolutionarily conserved histones. 
Citation: Lakhal S, Duthie MS (2017) In Silico Identification of B-Cell Epitopes of Leishmania infantum Recombinant Histone Shared with Human Sera Stably Living in Area Where Leishmania Species Does Perpetuate. Next Generat Sequenc \& Applic 4: 146. doi: $10.4172 / 2469-9853.1000146$

Page 2 of 7

\section{Patients and Methods}

\section{Serum samples}

All analyses were conducted in compliance with the current laws of Tunisia, the country where the study was conducted. The study was retrospective, so no other requirements were required relating to the subjects from whom the samples originated. The ANA positive sera analyzed in this work are part of clinical practice-based laboratory specimens of the Laboratory of Clinical Immunology, Pasteur Institute of Tunis, Tunisia.

Three panels of sera were screened, with all samples provided from Tunisian subjects. The first panel $(n=42)$ served as a control group (1) and was prepared from healthy pregnant women living in North Tunisia, an area where $L$. infantum is endemic. These sera were initially collected for the purpose of defining their status with respect to colonization (or not) by $T$. gondii through the presence of Toxoplasma gondii-binding antibodies. All of these sera had ANA titers $<1 / 80$ and did not possess extractable nuclear antigens (ENA)-binding autoantibodies as determined by indirect immunofluorescence on Hep-2 cells (DiaSorin Inc., USA).

A second panel $(n=42)$ served as an auxilliary control group (2) formed from samples of individuals from both VL-endemic and nonendemic regions. It was kindly provided by national blood transfusion center (NBTC) and was prepared from voluntary, healthy adults blood donors.

Sera in panel also had ANA titers $<1 / 80$ and did not possess extractable nuclear antigens (ENA)-binding autoantibodies as determined by indirect immunofluorescence on Hep- 2 cells. The third panel $(n=80)$ was prepared from healthy adults considered at risk of developing Systemic Lupus Erythematosus (SLE) episodes. Each sample in this panel was assessed by the Clinical Immunology Laboratory of Institut Pasteur de Tunis for the presence of ANAbinding antibodies. Each presented ANA titers $\geq 80$ by indirect immunofluorescence on Hep-2 cells.

\section{Antigens}

Soluble Leishmania Antigen/SLA was prepared from L. infantum promastigotes after ultrasonic treatment, as described previously [22,23]. Crude Leishmania Histones (CLH) were extracted according to a standard approach of histone protein isolation [24] with some modifications $[23,24]$. rK39, a recombinant product of the 39 amino acid repeats found in a kinesin-like gene of viscerotropic Leishmania species [25], was kindly provided by Dr. Steven G. Reed, infectious disease research institute (IDRI), Seattle, WA. L. infantum/chagasi derived recombinant proteins $\mathrm{rH} 2 \mathrm{~A}, \mathrm{rH} 2 \mathrm{~B}$, and $\mathrm{rH} 4$ were kindly provided by Dr. Manuel Soto Manuel Centro de Biologóa Molecular "Severo Ochoa", Facultad de Ciencias, Universidad Autonoma de Madrid, Madrid, Spain [11-14].

\section{Detection of antigen-specific antibodies by enzyme linked immunosorbent assay (ELISA)}

Antibodies against CLH, SLA and rK39 were detected by ELISA, as described previously [23]. Antibodies against $\mathrm{rH} 2 \mathrm{~A}, \mathrm{rH} 2 \mathrm{~B}$ and $\mathrm{rH} 4$ were detected as follows. ELISA were optimized for antigen coating concentration $(\mathrm{rH} 2 \mathrm{~A}[10 \mu \mathrm{g} / \mathrm{ml}], \mathrm{rH} 2 \mathrm{~B}[5 \mu \mathrm{g} / \mathrm{ml}]$ and $\mathrm{rH} 4[5 \mu \mathrm{g} / \mathrm{ml}])$, each serum was diluted $1 / 100$ and antibodies detected with horseradish peroxidase-conjugated anti-human IgG diluted 1/10,000.
The optical density (OD) of each well was read at dual wavelengths (492 $\mathrm{nm}$ and $620 \mathrm{~nm}$ ) by ELISA plate reader (Anthos 2020, Biochrom Ltd., United Kingdom). For each serum, the mean OD value was calculated from duplicate wells. The cut off threshold values for positive responses against each antigen were defined as the mean OD plus two standard deviations of the OD obtained with sera from control group (1). OD thresholds values for SLA, rK39 and CLH were set to $0.912,0.394$ and 0.339 , respectively. The cut off values for $\mathrm{rH} 2 \mathrm{~A}$, $\mathrm{rH} 2 \mathrm{~B}$, and $\mathrm{rH} 4$ were $0.559,0.653$, and 0.251 , respectively.

\section{Detection of subjects at risk of developing SLE}

Serum ANA levels were measured using ANA screen 9 kit (Euroimmun, Lübeck, De) using the manufacturer's suggested cut-off to define positive responses [1]. Then, antibodies recognizing Extracted Nuclear Autoantigens (ENA) (consisting of Sm, RNP, SS-A, SS-B, Scl-70, RNP, Jo-1, centromeres, and ribosomal $P$ proteins) were detected with the ENA Profile 1 Euroline (Euroimmun, Lübeck, De).

Mammalian histones-binding antibodies were screened with an anti-histones ELISA (IgG) (Euroimmun, Lübeck, De), with values $>20$ $\mathrm{IU} / \mathrm{mL}$ considered as positive. These assays allow detecting antibodies that bind the following antigens: PCNA, PM-Scl, ribosomal P proteins, $\mathrm{nRNP} / \mathrm{Sm}$. The cutoff was $\geq 20 \mathrm{RU} / \mathrm{ml}$. Native double-stranded DNA (dsDNA) binding antibodies were detected by indirect immunofluorescence using Crithidiae luciliae as substrate (Euroimmun, Lübeck, De). Titers $\geq 20$ were considered positive.

\section{Bioinformatics analysis}

To identify conserved and divergent amino acid sequences between Leishmania infantum histones H2A (access number: CAD11893.1), H2B (access number: CAA73826.1), H3 (access number: CAA54693), and their human homologs we used the BLAST server NCBI (http:// blast.ncbi.nlm.nih.gov).

Each blast focused on Homo sapiens (taxid: 9606). The selected sequences identical to histone proteins of $L$. infantum were histone H2A type 1-A (accession number NP_734466.1), histone H2B type 1-L (accession number NP_003510.1), and histone H4 (access number NP_003529.1). Protein sequence alignment of human and Leishmaniachagasi histones were rendered with T-COFFEE.

(M-Coffee; http://tcoffee.crg.cat/apps/tcoffee/do:mcoffee) and EMBOSS Needle EMBL-EBI (http://www.ebi.ac.uk/Tools/psa/ emboss_needle/).

Antigenic peptides were predicted with an accuracy of $75 \%$ using the method described by Burns [26], with the software available at http://imed.med.ucm.es/Tools/antigenic.pl (Complutense University of Madrid). Using this software sequence peptide segment is considered significant if it has a minimum of 8 amino acids.

\section{Statistical analysis}

Statistical analysis was performed using Prism Version 5 software (Graph Pad Prism Inc., San Diego, CA). Differences in antibody levels between the control groups were assessed using the Kruskal-Wallis test with Dunn post-test. Differences between two groups were compared with Mann Whitney of t-test (non-parametric tests). A p-value of $<0.05$ was considered significant. 
Citation: Lakhal S, Duthie MS (2017) In Silico Identification of B-Cell Epitopes of Leishmania infantum Recombinant Histone Shared with Human Sera Stably Living in Area Where Leishmania Species Does Perpetuate. Next Generat Sequenc \& Applic 4: 146. doi: $10.4172 / 2469-9853.1000146$

Page 3 of 7

\section{Results}

\section{Circulating antibodies against host antigens}

The sera prepared from subjects at risk of developing SLE episodes, as indicated by the presence of ANA-binding autoantibodies $(n=80$, ANA titers $\geq 80$ ) were tested for the presence of ENA-binding antibodies. All of the ANA-positive samples had antibodies against one or more ENA, particularly SS-A, SS-B, Ro52, RNP, Anti-Jo, Sm and NUC, as well as mammalian histones (Table 1).
Ten percent ( 8 of 80 ) of the ANA-positive sera also had detectable anti-DNA antibodies, while $37.5 \%$ (30 of 80 ) had antibodies that bound mammalian histones. In contrast, no ENA- or dsDNA-binding antibodies were detected in either of the control groups. Together, these data indicate the broad range of antibodies against host antigens in individuals at risk of developing autoimmune disease.

\begin{tabular}{|c|c|c|c|c|}
\hline Patient code & ANA & dsDNA & ENA (ND) & Number of cases with absorbance mammalian \\
\hline & IF & & & Histones value ( $>20 \mathrm{lU} / \mathrm{mL}$ ) \\
\hline P2, P9, P32, P51, P71, P78 & + & + & ND & $\mathrm{N}=6$ \\
\hline $\begin{array}{l}\text { P11, P12, P14, P22, P26, P34, P35, P36, P39, } \\
\text { P46, P49, P50, P56, P58, P63, P69, P70, P73, } \\
\text { P77, P79. }\end{array}$ & + & - & ND & $\mathrm{N}=20$ \\
\hline P18, P44, P52, P68 & + & - & $\begin{array}{l}\text { SS-A, RNP, } \quad \text { Sm; } \\
\text { NUC; } \\
\text { Ro5-A; }\end{array}$ & $\mathrm{N}=4$ \\
\hline $\begin{array}{l}\text { P1, P3, P4, P5, P6, P7, P8, P10, P15, P16, P19, } \\
\text { P20, P23, P24, P25, P27, P28, P29, P31, P33, } \\
\text { P37, P38, P40, P41, P45, P47, P48, P55, P59, } \\
\text { P60, P61, P64, P65, P66, P67, P74, P75, P76. }\end{array}$ & + & - & ND & $\mathrm{N}=38$ \\
\hline P17, P30, P42, P43, P54, P62, P72, P80 & + & - & $\begin{array}{l}\text { SS-A, SS-B, Ro52; } \\
\text { SS-A, SS-B, Ro52; } \\
\text { SS-A, Anti-Jo, Ro52; } \\
\text { SS-A, Ro52; RNP, } \\
\text { Ro52; Scl 70; SS-A, } \\
\text { SS-B, Ro52; SS-A, } \\
\text { SS-B+/-, Ro52 }\end{array}$ & $\mathrm{N}=8$ \\
\hline P21 & + & + & SS-A, SS-B & $N=1$ \\
\hline P53, P57 & + & + & ND & $\mathrm{N}=2$ \\
\hline
\end{tabular}

Table 1: Profile of antibodies against extractable nuclear antigens (ENA) and mammalian histones in samples that were ANA-positive.

\section{Circulating antibodies against Leishmania antigens}

The potential overlap of autoimmune diseases and VL presents a situation of confounding diagnoses if cross-reactive tests are used. We first explored the possibility that samples from individuals at risk of developing SLE due to the presence of antibodies against human histones had antibodies that also reacted with Leishmania histones. To determine the apparent $L$. infantum infection status of our subjects, sera were also evaluated for antibodies against a crude mixture of $L$. infantum antigens (SLA). Results indicate that $40 \%$ of SLE patients, but only 1 sample from the VL-endemic region and none from the nonendemic region, possessed antibodies that could bind SLA (Figure 1A).

While none of the control samples, be they from VL-endemic or non-endemic regions, reacted with $\mathrm{CLH}, 30 \%$ of the SLE patients presented with positive responses against CLH (Figure 1B). When samples were assessed against the much more refined recombinant rK39 antigen, the proportion of SLE patients indicated to have $L$. infantum infection dropped to $13.75 \%$ (Figure 1C). Within the panel of sera from individuals at risk of SLE, 30\% (24 of 80 ) had detectable antibodies against Crude Leishmania Histones (CLH).

Of these 15 anti-CLH positive sera, 9 contained SLA-binding antibodies and 6 contained rK39-binding antibodies (Table 2). Moreover, these 15 sera also relatively high levels of antibodies against mammalian histones, as well as a range of ENA-binding autoantibodies (Table 2). Together, these data support our hypothesis that individuals at risk of developing autoimmune diseases have antibodies that crossreact with $L$. infantum antigens.

\section{Antibody responses against Leishmania histones}

To further explore this likely cross-reactivity, we investigated responses against recombinantly-expressed $\mathrm{H} 2 \mathrm{~A}, \mathrm{H} 2 \mathrm{~B}$ and $\mathrm{H} 4$ that were generated using Leishmania gene sequences. 
Citation: Lakhal S, Duthie MS (2017) In Silico Identification of B-Cell Epitopes of Leishmania infantum Recombinant Histone Shared with Human Sera Stably Living in Area Where Leishmania Species Does Perpetuate. Next Generat Sequenc \& Applic 4: 146. doi: $10.4172 / 2469-9853.1000146$

Page 4 of 7

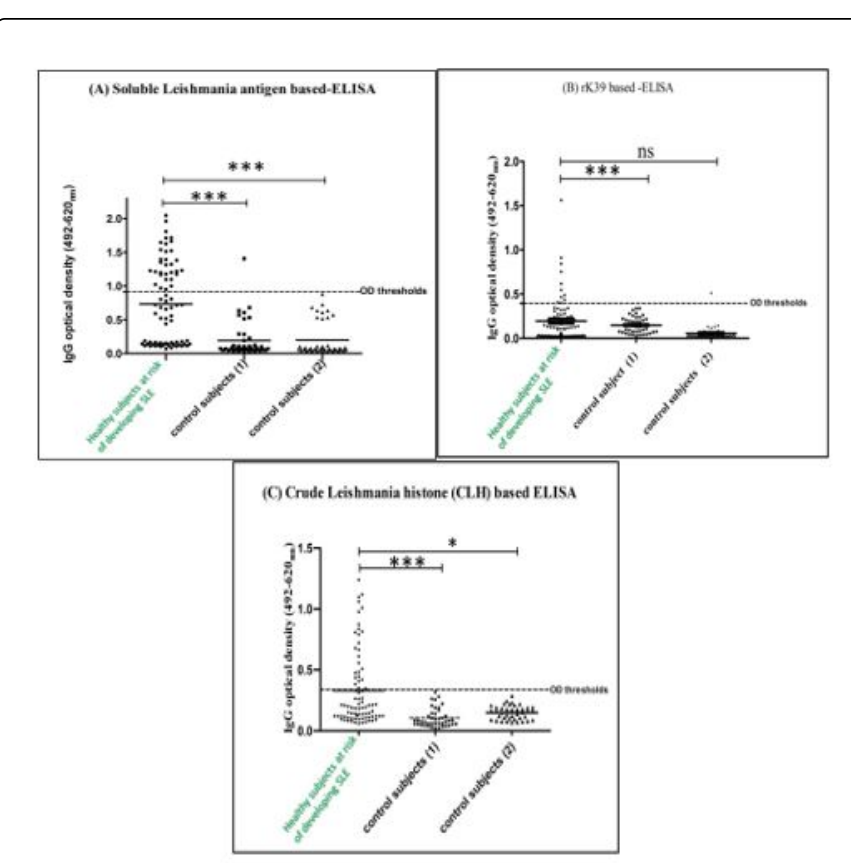

Figure 1: Antigen-specific antibody responses of study population. Sera from subjects either at risk of developing SLE $(n=80)$, or living in VL-endemic $(n=42)$ or non-endemic $(n=42)$ areas were tested for the presence of antibodies against (A) SLA, (B) CLH and (C) rK39, respectively. The threshold for positive results is indicated by the dotted line, and was calculated from control group (1). Each point represents results from one samples and the solid lines represent the median values for each group. Statistical analysis was conducted using Kruskal-Wallis test with Dunn's posttest for multiple comparisons. ${ }^{*} \mathrm{p}<0.05,{ }^{* *} \mathrm{p}<0.001{ }^{\star *}, \mathrm{p}<0.0001$.

\begin{tabular}{|l|l|l|l|l|l|l|l|}
\hline $\begin{array}{l}\text { Sample } \\
\text { code }\end{array}$ & $\begin{array}{l}\text { Anti-mammalian } \\
\text { histones (IU/mL) }\end{array}$ & CLH $^{1}$ & rH2A & rH2B & rH4 & SLA & K39 \\
\hline P2 & 59.038 & 0.437 & 0.926 & 0.646 & 0.263 & 1.394 & 0.03 \\
\hline P9 & 123.654 & 0.611 & 0.876 & 0.394 & 0.081 & 0.802 & 0.174 \\
\hline P12 & 238.462 & 0.666 & 0.365 & 0.654 & 0.187 & 1.166 & 0.241 \\
\hline P14 & 79.423 & 0.475 & 0.606 & 0.445 & 0.213 & 0.967 & 1.561 \\
\hline p22 & 407.308 & 0.679 & 0.776 & 0.824 & 0.219 & 1.227 & 0.029 \\
\hline p26 & 106.731 & 0.446 & 0.843 & 0.2 & 0.209 & 1.312 & 0.03 \\
\hline p32 & 188.462 & 0.408 & 0.9 & 0.211 & 0.205 & 1.013 & 0.91 \\
\hline p35 & 21.154 & 0.791 & 0.244 & 0.956 & 0.086 & 1.716 & 0.336 \\
\hline p43 & 407.307 & 0.509 & 0.39 & 0.669 & 0.093 & 0.19 & 0.123 \\
\hline p44 & 48.269 & 0.558 & 0.359 & 0.302 & 0.185 & 1.212 & 0.753 \\
\hline p46 & 22.308 & 0.482 & 0.243 & 0.262 & 0.107 & 0.161 & 0.021 \\
\hline p49 & 72.308 & 1.119 & 0.317 & 0.288 & 0.129 & 1.218 & 0.843 \\
\hline p71 & 295.769 & 1.01 & 0.317 & 0.18 & 0.327 & 0.126 & 0.129 \\
\hline p73 & 100.577 & 0.978 & 0.282 & 0.247 & 0.201 & 0.103 & 0.015 \\
\hline
\end{tabular}

Figure 2: Anti-histone responses of subjects. Serum samples that demonstrated the presence of antibodies against crude histones $(\mathrm{CLH})$ were further evaluated for the presence of antibodies against (A) $\mathrm{rH} 2 \mathrm{~A},(\mathrm{~B}) \mathrm{rH} 2 \mathrm{~B}$ and (C) $\mathrm{rH} 4$, respectively. Sera from $\mathrm{X}$ healthy individuals at risk of developing SLE and Y healthy subjects living in a VL-endemic region (control 1) were assessed. The threshold to distinguish negative and positive samples is indicated by the dotted line, and was calculated from control group (1). Each point represents results from one samples and the solid lines represent the median values for each group. Statistical analysis was conducted using Mann-Whitney test of $\mathrm{t}$-tests (and non-parametric tests) for comparisons. ${ }^{\star} \mathrm{p}<0.05,{ }^{\star *} \mathrm{p}<0.001{ }^{\star * \star} \mathrm{p}<0.0001$.

Of the anti-CLH sera evaluated, $\mathrm{rH} 2 \mathrm{~A}$ yielded a positive response in $63.63 \%$ (Figure 2A), rH2B yielded a positive response in $59.09 \%$ (Figure $2 \mathrm{~B}$ ) and $\mathrm{rH} 4$ yielded a positive response in all (100\%) (Figure 2C). Whereas responses against CLH in control subjects (1) were all below OD 0.4 (Figure 1), some subjects from the same group showed stronger responses against single proteins representing only a fraction of CLH (Figure 2).These data validate the in-silico predictions and 
Citation: Lakhal S, Duthie MS (2017) In Silico Identification of B-Cell Epitopes of Leishmania infantum Recombinant Histone Shared with Human Sera Stably Living in Area Where Leishmania Species Does Perpetuate. Next Generat Sequenc \& Applic 4: 146. doi: $10.4172 / 2469-9853.1000146$

Page 5 of 7

indicate that the human histones are immunologically cross-reactive with Leishmania histones.

\section{Prediction of antibody-binding epitopes within histones}

Genetic analysis indicates that the L. infantum H2B, H2A and H4 proteins and their human homologs are highly conserved. To make in silico predictions of the antibody-binding epitopes shared between $L$. infantum H2A (CAD11893.1), H2B (CAA73826.1) and $\mathrm{H} 4$ (CAA74210.1) and their respective human counterparts (histone $\mathrm{H} 2 \mathrm{~A}$ type 1-A, NP_734466.1; histone H2B type 1-L, NP_003510.1 and histone H4, NP_003529.1), blasts were conducted against human proteins (Homo sapiens, taxid: 9606).

The conserved region of recombinant $\mathrm{H} 2 \mathrm{~A}, \mathrm{H} 2 \mathrm{~B}$ and $\mathrm{H} 4$ exhibits $42.1 \%, 23.8 \%$ and $52.9 \%$ identity relative to their human histone homologs, respectively. Interestingly, H2A displays 4 segmental epitopes with highly conserved amino acid sequences (residues 17-29, 45-72, 77-90, 96-119) (Figure 3), H2B displays 5 segmental epitopes with highly conserved sequence (residues 8-17, 23-35, 49-56, 61-71, 82-97) (Figure 4), and H4 displays 2 segmental epitopes (residues 16-23 ( $<8$, non-sig), 42-67, 80-89, 43-49 ( $<8$, non-sig) (Figure 5). These predictions suggest cross-reactivity between autoimmune and $L$. infantum-infected samples is likely.

\begin{tabular}{|c|c|c|}
\hline CAD11893.1 & 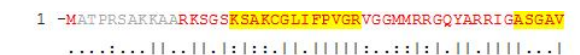 & 49 \\
\hline $\mathrm{NP}_{-} 734466.1$ & 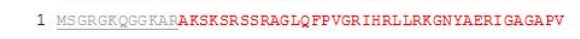 & 50 \\
\hline CAD1 1893.1 & 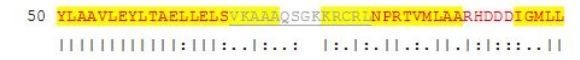 & 99 \\
\hline NP_734466.1 & 51 YLAAVLEYLTAEI LELAGNA SRDN--KRTRII PRHLQLAIRNDEELNRLI & 98 \\
\hline $\begin{array}{l}\text { CAD1 } 1893.1 \\
\text { NP_734466.1 }\end{array}$ & 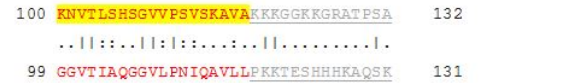 & \\
\hline \multicolumn{3}{|c|}{$\begin{array}{l}\text { Figure 3: Alignment of amino-acid sequences corresponding to the } \\
\text { ORF H2A protein of Leishmania infantum. The alignment was } \\
\text { performed with the EMBOSS software Needle EMBL-EBI. The } \\
\text { divergent regions of L. infantum } \mathrm{H} 2 \mathrm{~A} \text { are colored in gray letters and } \\
\text { underlined. Similar areas of amino acids are colored red. The } \\
\text { antigenic peptides are highlighted in yellow letters marked. } \\
\text { (|): indicates identical amino acids. (.): indicates similar amino } \\
\text { acids. Gaps were inserted to achieve maximum similarity and are } \\
\text { indicated by dashed lines. }\end{array}$} \\
\hline
\end{tabular}

\section{Discussion}

L. infantum is endemic in northern Tunisia and many Tunisians therefore reside in regions where zooanthropophilic blood-feeding sand flies and Leishmania/L. infantum co-exist. Although the burden of VL in North Africa is mainly in children aged between 1 and 4 years of age, VL can present clinical and laboratory autoimmune manifestations including arthralgia, cutaneous vasculitis, increased titers of rheumatoid factor (RF) and antinuclear antibodies (ANA) [27-29]. VL patients may initially be misdiagnosed as having an autoimmune disease such as SLE or autoimmune hepatitis, leading to the possibility that they could be treated with immunosuppressive drugs with fatal consequences [30].

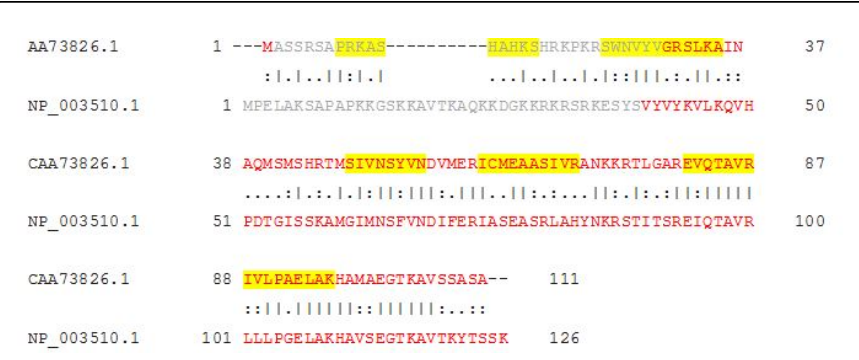

Figure 4: Alignment of amino-acid sequences corresponding to the ORF $\mathrm{H}_{2} \mathrm{~B}$ protein of Leishmania infantum. The alignment was performed with the EMBOSS software Needle EMBL-EBI. The divergent regions of $L$. infantum $\mathrm{H} 2 \mathrm{~B}$ are colored in gray letters and underlined. Similar areas of amino acids are colored red. The antigenic peptides are highlighted in yellow letters marked. (|): indicates identical amino acids. (.): indicates similar amino acids. Gaps were inserted to achieve maximum similarity and are indicated by dashed lines.

\begin{tabular}{|c|c|c|c|}
\hline CAA 74210.1 & 1 & 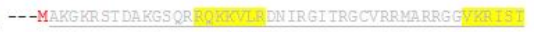 & 47 \\
\hline NP_-003529.1 & & 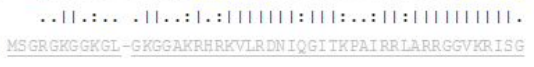 & 49 \\
\hline CAA74210.1 & 48 & 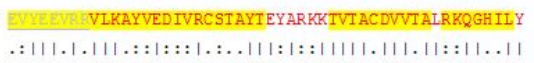 & 97 \\
\hline NP_003529.1 & 50 & IIYEETR VVLKVF LENVIRDAVTYTEHAKRKTVI AMDVVYALKRQGRTLY & 99 \\
\hline CAA74210.1 & & $\begin{array}{l}\text { GYA- } \quad 100 \\
1:\end{array}$ & \\
\hline NP_-003529.1 & 100 & GFGG & \\
\hline
\end{tabular}

Figure 5: Alignment of amino-acid sequences corresponding to the ORF $\mathrm{H} 4$ protein of Leishmania infantum. The alignment was performed with the EMBOSS software Needle EMBL-EBI. The divergent regions of $L$. infantum $\mathrm{H} 4$ are colored in gray letters and underlined. Similar areas of amino acids are colored red. The antigenic peptides are highlighted in yellow letters marked. (|): indicates identical amino acids. (.): indicates similar amino acids. Gaps were inserted to achieve maximum similarity and are indicated by dashed lines.

Indeed, several reports have described VL patients as being misdiagnosed with an autoimmune disease and then receiving immunosuppressive therapy, particularly anti-tumor necrosis factor agents [31]. In this study, we assessed the likelihood of confounding diagnoses of autoimmune disease and VL/L. infantum infection. Serum samples were evaluated for antibody responses against a classical panel of SLE-associated autoantigens and two antigens used for diagnosis of VL (crude soluble Leishmania antigen (SLA) and the defined rK39 protein). In addition, we assessed crude mixtures of Leishmania histones (CLH) and the related single recombinant histones $\mathrm{rH} 2 \mathrm{~A}, \mathrm{rH} 2 \mathrm{~B}$ and $\mathrm{rH} 4$ derived from $L$. infantum [27].

Fever, pancytopenia, splenomegaly, hyper-gammaglobulinemia, production of autoantibodies and complement consumption are some of the overlapping features between VL and SLE, presenting a serious problem for differential diagnosis [32]. As with rheumatic disease, VL patients have high levels of circulating immune complexes and autoantibodies such as rheumatoid factor, hyper-gammaglobulinemia and antinuclear antibodies (ANA) observed in systemic autoimmune 
Citation: Lakhal S, Duthie MS (2017) In Silico Identification of B-Cell Epitopes of Leishmania infantum Recombinant Histone Shared with Human Sera Stably Living in Area Where Leishmania Species Does Perpetuate. Next Generat Sequenc \& Applic 4: 146. doi: $10.4172 / 2469-9853.1000146$

Page 6 of 7

diseases in particular SLE. Autoantibodies against histones have also been detected in several autoimmune disorders like inflammatory arthritis, leukocytoclastic vasculitis and autoimmune hepatitis, primary biliary cirrhosis and SLE [33]. While the detection of ANA in human serum is an important screening tool for SLE and indirect immunofluorescence (IIF) is the gold method for ANA testing [34], histones have recently been indicated to be involved in the pathogenesis of SLE. In particular, antibodies specific to $\mathrm{H} 1$ and $\mathrm{H} 2 \mathrm{~B}$ have been detected [35], and our data confirm this.

We previously tested nucleosomal histones which have been extensively employed in ELISA against sera from human and canine VL. In addition, CLH-based ELISA showed an excellent ability to discriminate between VL cases and healthy controls (97.6\% sensitivity and $100 \%$ specificity). However, humoral response generated in sera from subjects at risk of developing SLE episodes cross-reacts with the parasitic Crude Leishmania histone $[23,25]$. In fact, the native CLHbased ELISA was revealed more accurate than single recombinant histone-based ELISA in some subjects from the same control group prepared from healthy pregnant women living in North Tunisia, an area where $L$. infantum is endemic and was more specific. Leishmania histone single protein fractions, specifically those involved in immune response and the production of the corresponding recombinant proteins in Eukaryotic Protein Expression system with Leishmania tarentolae could explain this result. In fact, this expression system allows eukaryotic protein folding and posttranslational modifications of target proteins, which may offer more specific and sensitive tools for VL diagnosis.

Recombinant $L$. infantum histones $\mathrm{rH} 2 \mathrm{~A}, \mathrm{rH} 2 \mathrm{~B}, \mathrm{rH} 3$, and $\mathrm{rH} 4$, have shown serodiagnostic potential for both human and canine VL [11-14]. In these studies histones presented a high specificity and sensitivity and were suggested as useful antigens for VL serodiagnosis [15]. The use of histones in the diagnosis of VL in subjects at risk of developing autoimmune complications such as SLE episodes was not defined. Recombinant histone-based ELISA revealed specificity but little sensitivity, especially when recombinant antigens were evaluated for CVL diagnosis (sensitivities of 63.63\%, 59.09\%, and $100 \%$ for $\mathrm{H} 2 \mathrm{~A}, \mathrm{H} 2 \mathrm{~B}$ and $\mathrm{H} 4$, respectively). All recombinant proteins $(\mathrm{H} 2 \mathrm{~A}, \mathrm{H} 2 \mathrm{~B}$ and $\mathrm{H} 4)$ were recognized by sera from subjects at risk of developing SLE episodes; $\mathrm{rH} 4$ presented the best performance in terms of sensitivity and specificity, being superior to $\mathrm{H} 2 \mathrm{~A}$ and $\mathrm{H} 2 \mathrm{~B}$ in the diagnosis of VL.

The antigenicity of recombinant histone proteins in general and that of rH4 in particular is not surprising in sera from subjects at risk of developing SLE episodes. Indeed, genomic analysis indicated that the conserved region of recombinant H4 exhibits high identity (52.9\%) with human histones and displays 2 segmental epitopes with highly conserved amino acid sequences (residues 42-67, 80-89). Although $\mathrm{H} 2 \mathrm{~A}$ and $\mathrm{H} 2 \mathrm{~B}$ were less conserved, identity was still $42.1 \%$ to $23.8 \%$ relative to human histones, respectively. Four segmental epitopes were indicated in H2B (residues 17-29, 45-72, 77-90, 96-119) and 5 segmental epitopes were indicated in $\mathrm{H} 2 \mathrm{~A}$ (residues 8-17, 23-35, 49-56, $61-71,82-97)$. These in silico predictions suggest that anti-histone antibodies in sera from subjects at risk of developing SLE episodes might recognize conserved regions within the parasite histones. Therefore, it is likely that the reaction of 4 sera against $\mathrm{rH} 4,9$ against $\mathrm{rH} 2 \mathrm{~A}$ and 4 against $\mathrm{rH} 2 \mathrm{~B}$ is a consequence of cross-reactivity.

Some of VL manifestations are associated with immune responses against Leishmania that mimic autoimmune diseases, autoimmune phenomena are common in leishmaniasis and might be due to a release of host antigens that are released during tissue destruction by these protozoa. Through such mechanism $L$. infantum infection may promote autoreactivity and consequently, production of autoantibodies. Other etio-pathogenic hypotheses are that these manifestations can be related to polyclonal B-cell activation, altered or reduced regulatory and suppressor $\mathrm{T}$ cell functions [27,28] or molecular mimicry between Leishmania antigens and host antigens $[28,29]$. Histone proteins $\mathrm{H} 2 \mathrm{~A}, \mathrm{H} 2 \mathrm{~B}$, and $\mathrm{H} 4$ play an important role in DNA packaging and, since no cross reactivity of VL sera with mammalian histones was observed, have been reported to induce a humoral response specific for Leishmania histones [11-15].

Our data indicate that in the reverse situation, circulating antibodies in many of the SLE at-risk individuals recognized parasite histones. This compromised the specificity of SLA-based ELISA, providing many false-positive results (38). Examination of the CLH mixture which is expected to contain nucleosomal Leishmania histones $\mathrm{H} 2 \mathrm{~A}, \mathrm{H} 2 \mathrm{~B}$ and $\mathrm{H} 4$ suggested these to be a source of the crossreactivity. Analyses of an additional panel of sera from diagnosed patients for SLE are required/necessary to further verify the conclusions. For the purposes of diagnosing VL, however, it is therefore important to note that the rK39 antigen was found to be more specific than the use of histones.

\section{Acknowledgments}

We would like to thank Dr. Steven G. Reed, Infectious Disease Research Institute (IDRI), Seattle, WA, for providing recombinant K39 of $L$. infantum. We thank M. Soto, Universidad Autónoma de Madrid, Spain, for providing recombinant $\mathrm{H} 2 \mathrm{~A}, \mathrm{H} 2 \mathrm{~B}$, and $\mathrm{H} 4$ of $L$. infantum/ chagasi.

\section{References}

1. Ready PD (2014) Epidemiology of visceral leishmaniasis. Clin Epidemiol 6: 147-154.

2. Singh OP, Hasker E, Sacks D, Boelaert M, Sundar S (2014) Asymptomatic Leishmania infection: A new challenge for Leishmania control. Clin Infect Dis 58: 1424-1429.

3. Von Stebut E (2015) Leishmaniasis. J Dtsch Dermatol Ges 13: 191-200.

4. Pace D (2014) Leishmaniasis. J Infect 69: S10-18.

5. Srivastava P, Dayama A, Mehrotra S, Sundar S (2011) Diagnosis of visceral leishmaniasis. Trans R Soc Trop Med Hyg 105: 1-6.

6. Srividya G, Kulshrestha A, Singh R, Salotra P (2012) Diagnosis of visceral leishmaniasis: Developments over the last decade. Parasitol Res 110: 1065-1078.

7. Badaró R, Benson D, Eulálio MC, Freire M, Cunha S (1996) rK39: A cloned antigen of Leishmania chagasi that predicts active visceral Leishmaniasis. J Infect Dis 173: 758-761.

8. Maalej IA, Chenik M, Louzir H, Salah AB, Bahloul C, et al. (2003) Comparative evaluation of ELISAs based on ten recombinant or purified Leishmania antigens for the serodiagnosis of Mediterranean visceral leishmaniasis. Am J Trop Med Hyg 68: 312-320.

9. Galaï Y, Chabchoub N, Ben-Abid M, Ben-Abda I, Ben-Alaya-Bouafif N, et al. (2011) Diagnosis of mediterranean visceral leishmaniasis by detection of Leishmania antibodies and Leishmania DNA in oral fluid samples collected using an Oracol device. J Clin Microbiol 49: 3150-3153.

10. Maia Z, Lírio M, Mistro S, Mendes CM, Mehta SR, et al. (2012) Comparative study of rK39 Leishmania antigen for serodiagnosis of visceral leishmaniasis: Systematic review with meta-analysis. PLoS Negl Trop Dis 6: el 484 . 
Citation: Lakhal S, Duthie MS (2017) In Silico Identification of B-Cell Epitopes of Leishmania infantum Recombinant Histone Shared with Human Sera Stably Living in Area Where Leishmania Species Does Perpetuate. Next Generat Sequenc \& Applic 4: 146. doi: $10.4172 / 2469-9853.1000146$

Page 7 of 7

11. Soto M, Requena JM, Gomez LC, Navarrete I, Alonso C (1992) Molecular characterization of a Leishmania donovani infantum antigen identified as histone H2A. Eur J Biochem 205: 211-216.

12. Soto M, Requena JM, Quijada L, García M, Guzman F (1995) Mapping of the linear antigenic determinants from the Leishmania infantum histone H2A recognized by sera from dogs with leishmaniasis. Immunol Lett 48 : 209-214.

13. Soto M, Requena J, Quijada L, Gomez L, Guzman F, et al. (1996) Characterization of the antigenic determinants of the Leishmania infantum histone $\mathrm{H} 3$ recognized by antibodies elicited during canine visceral leishmaniasis. Clin Exp Immunol 106: 454-461.

14. Soto M, Requena JM, Quijada L, Perez MJ, Nieto CG, et al. (1999) Antigenicity of the Leishmania infantum histones $\mathrm{H} 2 \mathrm{~B}$ and $\mathrm{H} 4$ during canine viscerocutaneous leishmaniasis. Clin Exp Immunol 2: 342-349.

15. Meddeb-Garnaoui A, Toumi A, Ghelis H, Mahjoub M, Louzir H, et al. (2010) Cellular and humoral responses induced by Leishmania histone $\mathrm{H} 2 \mathrm{~B}$ and its divergent and conserved parts in cutaneous and visceral leishmaniasis patients, respectively. Vaccine 28: 1881-1886.

16. Argov S, Jaffe C, Krupp M, Slor H, Shoenfeld Y (1989) Autoantibody production by patients infected with Leishmania. Clin Exp Immunol 76: 190-197.

17. Nozzi M, Del Torto M, Chiarelli F, Breda L (2014) Leishmaniasis and autoimmune diseases in pediatric age. Cell Immunol 292: 9-13.

18. Liberopoulos E, Kei A, Apostolou F, Elisaf M (2013) Autoimmune manifestations in patients with visceral leishmaniasis. J Microbiol Immunol Infect 46: 302-305.

19. Wallis PJ, Clark CJ, Ann Rheum Dis. (1983) Visceral leishmaniasis complicating systemic lupus erythematosus. Ann Rheum Dis 42: 201-202.

20. Granel B, Serratrice J, Swiader L, Gambarelli F, Daniel L, et al. (2000) Crossing of antinuclear antibodies and anti-Leishmania antibodies. Lupus 9: 548-550.

21. Aoun K, Kaaroud H, Hamzaoui S, Siala E, Kooli C, et al. (2004) Particularities of visceral leishmaniasis in adults not infected by HIV in Tunisia. Med Trop 64: 160-162.

22. Melby PC, Neva FA, Sacks DL (1989) Profile of human T cell response to Leishmanial antigens. Analysis by immunoblotting. J Clin Invest 83: 1868-1875.

23. Lakhal S, Mekki S, Ben-Abda I, Mousli M, Amri F, et al. (2012) Evaluation of an enzyme-linked immunosorbent assay based on crude
Leishmania histone proteins for serodiagnosis of human infantile visceral leishmaniasis. Clin Vaccine Immunol 19: 1487-1491.

24. Shechter D, Dormann HL, Allis CD, Hake SB (2007) Extraction, purification and analysis of histones. Nat Protoc 2: 1445-1457.

25. Lakhal S, Benabid M, Sghaier IB, Bettaieb J, Bouratbine A, et al. (2015) The sera from adult patients with suggestive signs of autoimmune diseases present antinuclear autoantibodies that cross-react with Leishmania infantum conserved proteins: crude Leishmania histone and Soluble Leishmania antigens. Immunol Res 61: 154-159.

26. Burns JM Jr, Shreffler WG, Benson DR, Ghalib HW, Badaro R, et al. (1993) Molecular characterization of a kinesin-related antigen of Leishmania chagasi that detects specific antibody in African and American visceral leishmaniasis. Proc Natl Acad Sci USA 90: 775-779.

27. Casato M, de Rosa FG, Pucillo LP, Ilardi I, di Vico B, et al. (1999) Mixed cryoglobulinemia secondary to visceral leishmaniasis. Arthritis Rheum 42: 2007-2011.

28. Voulgari P, Pappas G, Liberopoulos E, Elisaf M, Skopouli F, et al. (2004) Visceral leishmaniasis resembling systemic lupus erythematosus, Ann Rheum Dis 63: 1348-1349.

29. Galvão-Castro B, Sá Ferreira JA, Marzochi KF, Marzochi MC, Coutinho SG, et al. (1984) Polyclonal B cell activation, circulating immune complexes and autoimmunity in human American visceral leishmaniasis. Clin Exp Immunol 56: 58-66.

30. Kolaskar AS, Tongaonkar PC (1990) A semi-empirical method for prediction of antigenic determinants on protein antigens. FEBS Lett 10: $172-174$.

31. Jean-Benoît A, Capron L, Pouchot J (2010) Visceral leishmaniasis mimicking systemic lupus erythematosus. J Clin Rheumatol 16: 203-204.

32. Gabler C, Kalden JR, Lorenz HM (2003) The putative role of apoptosismodified histones for the induction of autoimmunity in systemic lupus erythematosus. Biochem Pharmacol 66: 1441-1446.

33. Pigott DM, Bhatt S, Golding N, Duda KA, Battle KE, et al. (2014) Global distribution maps of the leishmaniases. Elife.

34. Bates PA, Depaquit J, Galati EA, Kamhawi S, Maroli M, et al. (2015) Recent advances in phlebotomine sand fly research related to leishmaniasis control. Parasit Vectors 8: 131.

35. Boelaert M, Verdonck K, Menten J, Sunyoto T, van Griensven J, et al. (2014) Rapid tests for the diagnosis of visceral leishmaniasis in patients with suspected disease. Cochrane Database Syst Rev 6: CD009135. 